\title{
Insatisfação Corporal e Atividade Física em Adolescentes da Região Continental de Florianópolis ${ }^{1}$
}

\author{
Fernando Adami² \\ Universidade de São Paulo \\ Deivis Elton Schlikmann Frainer \\ Universidade Federal da Bahia \\ João Severo Santos \\ Fundação Universidade Federal do Tocantins \\ Tony Charles Fernandes \\ Universidade do Estado de Santa Catarina \\ Fernando Roberto De-Oliveira \\ Universidade Federal de Lavras
}

\begin{abstract}
RESUMO - A relação entre atividade física e insatisfação corporal é pouco explorada na literatura. Foi examinada a insatisfação corporal em relação com o nível de atividade física em estudantes adolescentes de escolas públicas de Florianópolis. A amostra foi de 242 estudantes, 109 meninos (14,6 $\pm 2,8$ anos) e 133 meninas (14,3 \pm 3 anos). Os instrumentos utilizados foram: questionário de atividades físicas habituais e escala de percepção de silhueta corporal. Quarenta e três por cento dos meninos e $29,4 \%$ das meninas são pouco ativos. Sessenta e nove por cento dos meninos e $76,7 \%$ das meninas estão insatisfeitos com sua silhueta corporal. Os meninos demonstram uma tendência em aumentar e diminuir sua silhueta, enquanto as meninas demonstram querer diminuir. Não foi encontrada relação entre satisfação com a silhueta corporal e nível de atividade física habitual. Investigar a relação entre atividade física e insatisfação corporal pode auxiliar em pesquisas futuras que verifiquem intervenções terapêuticas com atividade física no tratamento da insatisfação corporal.
\end{abstract}

Palavras chave: insatisfação corporal; atividade física; adolescentes; silhueta corporal.

\section{Body Dissatisfaction and Physical Activity in Adolescents from Florianópolis Continental Region}

\begin{abstract}
The relationship between physical activity and body dissatisfaction is little explored in literature. It was examined the body dissatisfaction and physical activity relationship among adolescents students of public schools from Florianópolis, Brazil. The sample was composed by 242 students, 109 boys (14,6 $\pm 2,8$ years) and 138 girls (14,3 \pm 3 years). The instruments used were a habitual physical activities questionnaire and a body silhouette's scale. Forty three per cent of girls and $29,4 \%$ of boys are insufficiently active. Sixty nine per cent of the boys and 76,7\% of the girls are unsatisfied with their body silhouette. Boys demonstrate a tendency of both increase and decrease their body silhouette, while girls demonstrate a tendency to decrease their body silhouette. No association was found between satisfaction with body silhoutte and habitual physical activity status. To investigate the relationship between physical activity and body dissatisfaction can be useful for future research that could be verified in the therapeutical interventions with physical activity for the body dissatisfaction treatment.
\end{abstract}

Key words: body dissatisfaction; physical activity; adolescents; body silhouette.

A insatisfação corporal faz parte de um componente da imagem corporal relacionada com as atitudes e avaliações do próprio corpo (Grabe \& Hide, 2006; Scagliusi \& cols., 2006). Dessa forma, a insatisfação corporal pode ser definida como a avaliação negativa do próprio corpo. Ela é diagnosticada por meio de figuras do corpo e partes dele (como barriga e quadris) e questionários com perguntas sobre peso corporal e aspectos relacionados com a aceitação corporal (Stice \& Shaw, 2002).

O período da puberdade é considerado crítico em relação à insatisfação corporal. Isso em parte é devido ao fato de que nessa idade há uma maior percepção por parte do adolescente em relação às influencias provenientes de pais, amigos e

1 Fonte financiadora - PAP/UDESC.

2 Endereço: Rua Émerson Ferrari, nº 28, Ap. 1304, Kobrasol, São José, Santa Catarina, SC, Brasil 88102-060.E-mail: adamifernando@uol.com.br mídia sobre questões relacionadas ao corpo (McCabe \& Ricciardelli, 2005; McCabe, Ricciardelli \& Finemore, 2002). Além disso, o aumento nas dimensões corporais também é determinante para a questão da aceitação do corpo (Bearman, Martinez \& Stice, 2006; McCabe \& Ricciardelli, 2004).

Nas meninas, estudos prospectivos têm demonstrado que os preditores da insatisfação corporal na adolescência envolvem pressão percebida para a adequação ao esteriótipo de magreza e o excesso de massa corporal (Stice \& Bearman, 2001; Stice \& Shaw, 2002; Stice \& Whitenton, 2002), sendo que estudos mais recentes têm encontrado variáveis como comparação social e suporte social (familiar e amigos) (Bearman \& cols., 2006; Jones, 2004). Nos meninos, poucos estudos têm sido feito para determinar preditores da insatisfação corporal, podendo-se destacar as variáveis internalização do ideal de musculosidade (Jones, 2004) e o suporte familiar (Bearman \& cols., 2006). 
Há diferenças marcantes bem estabelecidas na literatura entre meninos e meninas adolescentes quanto aos aspectos de ideal de beleza e massa corporal. Enquanto nas moças o ideal de beleza está amplamente vinculado à magreza, nos rapazes esse ideal está voltado para a quantidade de músculo (Jones, 2004). Dessa forma, as meninas têm uma tendência de desejar a perda de peso e uma forma corporal mais magra. De fato, parece existir uma ênfase sócio-cultural para que as mulheres tenham que se adequar a um ideal de atratividade que está imbricadamente associado à magreza (Mckinley, 1999; Smolak, Levine \& Thompson, 2001). Nos meninos, esse desejo parece ser dividido entre aqueles que querem aumentar e aqueles que querem diminuir seu peso ou forma corporal (Furnham, Badmin \& Sneade, 2002).

Assim, em relação à massa corporal, parece que enquanto nas moças o aumento de massa está associado com o aumento da insatisfação corporal (Jones, 2004; Stice \& Whitenton, 2002), nos rapazes essa relação parece ser quadrática, o que demonstra que indivíduos muito magros ou com excesso de peso têm maior risco de desenvolver insatisfação corporal (Presnel, Bearman \& Stice, 2004). Essa relação de massa corporal com insatisfação corporal está atrelada ao próprio ideal de beleza. Assim, o aumento de peso nas meninas está associado com um afastamento do ideal de magreza, enquanto a baixa massa corporal ou o excesso de peso nos meninos também está associado com o afastamento do ideal de beleza (Bearman \& cols., 2006).

Referente às conseqüências, a insatisfação corporal está associada com a etiologia de distúrbios alimentares, incluindo anorexia, bulimia e compulsão alimentar, principalmente nas mulheres (Beato-Fernández, Rodriguez-Cano, Belmonte-Llario \& Martinez-Delgado, 2004; Beato-Fernández \& Rodriguez-Cano, 2005; Stice \& Shaw, 2002; The Mcknight Investigators, 2003; Van Strien, Engels, Leeuwe \& Snoek, 2005). Outros quadros clínicos também estão associados com a insatisfação corporal, como tentativa de suicídio (Rodriguez-Cano, Beato-Fernández \& BelmonteLlario, 2006) e depressão (Johnson \& Wardle, 2005).

Poucos estudos têm demonstrado a relação existente entre insatisfação corporal e atividade física. McCabe e Ricciardelli (2004) encontraram que a dependência de atividade física é uma conseqüência da insatisfação corporal e serve como estratégia para a obtenção do ideal de beleza para as adolescentes. Outro estudo transversal (Furnham \& cols., 2002) encontrou que adolescentes que se exercitavam com o objetivo de controle de peso, atratividade e tônus muscular tinham maiores níveis de insatisfação corporal.

Nos meninos, nenhum estudo demonstrou essa relação. O que se sabe é que adolescentes envolvidos em esportes têm maiores incidências do uso de esteróides anabólicos (Wichstrom, 2006; Wichstrom \& Pedersen, 2001). A partir daí, se especula que a insatisfação corporal possa ser uma variável mediadora da relação entre prática esportiva e uso de esteróides anabólicos (McCabe \& Ricciardelli, 2004). Entretanto, não existem ainda evidências científicas para corroborar tal modelo.

Nesses estudos, a variável atividade física foi avaliada com base em questionários que não indicavam o nível de atividade física dos avaliados. Os questionários utilizados nesses estudos averiguavam os motivos relacionados à pratica de atividades físicas e/ou se os indivíduos praticavam ou não esporte. Esse tipo de questionário, contudo, pode dar informações muito enviesadas, bem como não refletem a quantidade de atividades físicas realizadas pelos indivíduos.
Dessa forma, é essencial a utilização de questionários que possam dar algum indicativo do nível de atividade física habitual realizada pelos avaliados quando se pretende fazer associações entre atividade física e qualquer outra variável.

Ainda em relação à atividade física, é ampla a literatura que trata das questões dos benefícios fisiológicos trazidos por ela. Dessa forma, diversos estudos têm demonstrado que a atividade física deve fazer parte tanto do tratamento quanto da prevenção de doenças crônicas não transmissíveis, como obesidade e as doenças cardiovasculares (Kimm \& cols., 2005; Pedersen \& Saltin, 2006). Entretanto, poucos estudos têm sido feitos na tentativa de verificar a influência da atividade física de um ponto de vista psicológico. Com a importância da insatisfação corporal em uma série de patologias, faz-se necessário o incremento de estudos que possam verificar a associação da atividade física com a satisfação com o próprio corpo.

O objetivo deste estudo é descrever a insatisfação corporal e sua relação com a atividade física em estudantes de escola públicas de uma região urbana do município de Florianópolis.

\section{Método}

\section{Participantes}

A amostra foi composta por 242 estudantes na faixa etária dos 11 aos 18 anos, sendo 109 meninos (14,6 \pm 2,8 anos; 55 $\pm 4,3 \mathrm{Kg} ; 162 \pm 14 \mathrm{~cm})$ e 133 meninas $(14,3 \pm 3$ anos; $50 \pm$ $12,2 \mathrm{Kg} ; 156 \pm 9 \mathrm{~cm})$ da $5^{\text {a }}$ série do ensino fundamental ao 3 ano do ensino médio, selecionados de forma não probabilística intencional (Thomas \& Nelson, 2002). A pesquisa foi feita com estudantes adolescentes de três escolas públicas situadas em um bairro do município de Florianópolis - SC. A caracterização e a distribuição da amostra conforme o turno escolar, a escolaridade (séries) e o nível socioeconômico dos escolares estão demonstrados na Tabela 1.

Tabela 1. Distribuição da amostra total dos escolares conforme o turno escolar, a escolaridade e o nível sócio-econômico.

\begin{tabular}{|c|c|c|}
\hline Estratificação & Freqüência & Percentual $(\%)$ \\
\hline Total & 242 & 100 \\
\hline \multicolumn{3}{|l|}{ Turno Escolar } \\
\hline Matutino & 86 & 35,5 \\
\hline Vespertino & 125 & 51,7 \\
\hline Noturno & 31 & 12,8 \\
\hline \multicolumn{3}{|c|}{ Ensino fundamental } \\
\hline 5- Série & 67 & 27,7 \\
\hline 6를ie & 37 & 15,3 \\
\hline 7a Série & 33 & 13,6 \\
\hline 8a Série & 19 & 7,9 \\
\hline \multicolumn{3}{|l|}{ Ensino médio } \\
\hline 1a Série & 43 & 17,8 \\
\hline 2- Série & 16 & 6,6 \\
\hline 3a Série & 27 & 7,0 \\
\hline \multicolumn{3}{|c|}{ Nível Socioeconômico } \\
\hline Classe A & 4 & 1,7 \\
\hline Classe B & 45 & 18,6 \\
\hline Classe C & 136 & 56,2 \\
\hline Classe D & 48 & 19,8 \\
\hline Classe E & 8 & 3,3 \\
\hline
\end{tabular}




\section{Instrumentos}

Figuras de silhueta corporal: Essa escala de silhueta corporal foi adaptada por Childress, Brewerton, Hodges e Farrel, (1993) a partir das figuras de silhueta corporal desenvolvidas por Stunkard e Sorensen (1983). Essa adaptação foi feita para que se permitisse o uso da escala em crianças e adolescentes. A escala consiste em oito figuras que representam diversas formas de contorno ou silhueta corporal, abrangendo a silhueta bem magra (silhueta 1) até a silhueta obesa (silhueta 8) (ver Figura 1). A escala de Stunkard e Sorensen foi validada no Brasil para a população de adultos (Scagliusi \& cols., 2006). Para a escala de silhuetas adaptadas por Childress, não existem ainda dados publicados sobre a validação desse instrumento. Nesse sentido, nosso grupo realizou um estudo preliminar na tentativa de obter evidências de validade iniciais desse instrumento (dados não publicados). A amostra constou dos mesmos indivíduos do presente estudo. Assim, os escolares deveriam escolher, entre as oito figuras de silhuetas, qual delas correspondia à sua silhueta corporal atual (cuja figura correspondia à silhueta que ela acreditasse possuir). Um grupo de três pesquisadores experientes no uso da escala também identificou a silhueta correspondente a cada escolar. O procedimento aconteceu numa sala separada, de forma individualizada com os escolares. Não houve diferenças entre a identificação feita pelos avaliadores e a silhueta corporal atual identificada pelos adolescentes. O coeficiente de correlação de Kendall entre as duas medidas foi significativa estatisticamente (valores de 0,70 e 0,55 para meninos e meninas, respectivamente, $p<0,05$ ).

Tal escala é considerada um indicador da imagem corporal e pode ser utilizada para verificação da insatisfação corporal. O grau de satisfação com a silhueta corporal (GSSc) foi obtido pela subtração entre silhueta corporal desejada (Scd - cuja figura correspondia à silhueta que a pessoa gostaria de possuir) e silhueta corporal atual (Sca), as quais foram identificadas pelo avaliado numa sala reservada, com uma explicação prévia do pesquisador. Esse GSSc demonstra a magnitude da insatisfação com a silhueta corporal; dessa forma, a magnitude pode ser positiva, caso o indivíduo queira aumentar a silhueta corporal, e pode ser negativa, caso o indivíduo queira diminuir a silhueta. Para a caracterização da insatisfação corporal, sem levar em conta indivíduos que queriam aumentar ou diminuir, o GSSc foi também utilizado em módulo [GSSc]. Foram considerados: a) insatisfeitos - indivíduos com [GSSc] $>0$ e b) satisfeitos - indivíduos com [GSSc] $=0$.

Questionário do nível de atividade física habitual. O questionário desenvolvido por Pate e cols. (1995) foi aplicado para verificar o nível de atividade física (NAF), sendo que esse instrumento oferece duas medidas: atividades físicas ocupacionais (AFO) e atividades físicas de lazer (AFL). As atividades ocupacionais seriam aquelas nas quais os indivíduos realizassem em seu cotidiano (como por exemplo, se ela costuma ir para a escola a pé ou de bicicleta ou até mesmo trabalhar em algum serviço pesado). Por sua vez, as atividades de lazer consistem naquelas relacionadas ao lazer e à pratica esportiva e de atividades físicas. A classificação do questionário se dá da seguinte forma: são considerados pouco ativos indivíduos com uma pontuação abaixo de 12, e suficientemente ativos indivíduos com pontuação acima de 12. Essa versão do instrumento mostrou ter boa aplicabilidade e fidedignidade entre adolescentes e adultos jovens (Nahas, 2003).

\section{Procedimentos}

O projeto de pesquisa foi aprovado pelo Comitê de Ética em Pesquisa da Universidade do Estado de Santa Catarina, o qual é credenciado no Comitê de Ética em Pesquisa (CONEP) do Ministério da Saúde, desde o ano de 1998. A coleta foi realizada em aproximadamente 60 dias, nos meses de setembro e outubro de 2004.

Para a coleta de dados, os adolescentes foram abordados no mínimo em dois dias, sendo na maioria dos casos em três dias. No primeiro dia, foram entregues os termos de consentimento livre e esclarecido para que os alunos pudessem levar aos pais, conforme normatização do Comitê de Ética da UDESC. Nos outros dias, foram coletados os dados de todos os indivíduos que trouxeram o consentimento assinado pelos pais. A coleta de dados aconteceu em dois momentos distintos: 1) em sala de aula, com orientação coletiva, onde os estudantes respondiam o questionário de atividade física; 2) numa sala distinta, onde os adolescentes respondiam o questionário de silhueta corporal.

\section{Análises estatísticas}

Para as análises, foram empregados os testes não paramétricos, Mann-Whitney (comparações entre sexos), Wilcoxon (comparações no mesmo sexo), e Qui-Quadrado, aceitando como nível mínimo de significância $p<0,05$. A escolha pela utilização da estatística não paramétrica se deu pelo fato de a maioria das variáveis não apresentarem distribuição normal no teste de KolmogorovSmirnov. O pacote estatístico utilizado foi o SPSS 10.0.

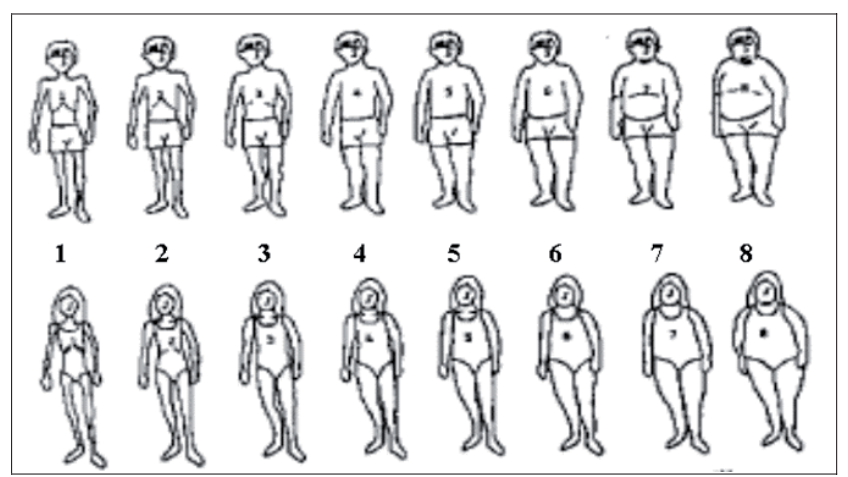

Figura 1. Escala de silhueta corporal de acordo com Childress e cols., (1993).

\section{Resultados}

A Tabela 2 caracteriza a amostra de acordo com a pontuação obtida no questionário de atividade física em termos de mediana e percentis, além de demonstrar a freqüência e percentual de acordo com a classificação da atividade física para meninos e meninas. Os dados apresentados evidenciam haver dependência entre a variável sexo e nível de atividade física. Dessa forma, a prevalência de meninas pouco ativas é significativamente superior a de meninos, respectivamente $43 \%$ e $29,4 \%\left(x^{2}=4,69 ; g l=1 ; p=0,033\right)$. Além disso, os meninos demonstraram ser mais ativos do que as meninas ( $U=5687,5 ; p=0,004)$. Parece que esse maior nível de atividade física é caracterizado por um maior nível de atividades físicas de lazer $(U=5410 ; p=0,001)$. 
Tabela 2. Mediana e percentis 25 e 75 (P25 e P75) do nível de atividade física (NAF), atividade física de lazer (AFL), atividade física ocupacional (AFO) e a frequiência da classificação quanto ao nível de atividade física, de acordo com o sexo.

\begin{tabular}{|c|c|c|c|c|c|c|}
\hline & Rapazes & & & Moças & & \\
\hline & Mediana & $P 25$ & $P 75$ & Mediana & $P 25$ & $P 75$ \\
\hline NAF & $15^{*}$ & 11 & 21 & 13 & 8 & 18 \\
\hline AFL & $9 *$ & 6 & 14 & 7 & 4 & 11 \\
\hline \multirow[t]{2}{*}{$\mathrm{AFO}$} & 7 & 4 & 7 & 5 & 3 & 7 \\
\hline & $n$ & $\%$ & & $n$ & $\%$ & \\
\hline Pouco Ativos & $32 \dagger$ & 29,4 & & 57 & 43 & \\
\hline Suficientemente Ativos & 77 & 70,6 & & 76 & 57 & \\
\hline
\end{tabular}

$* p<0,05$ entre sexos;

$\dagger p<0,05$ - Qui-Quadrado de Pearson

Os valores de mediana, moda e percentis 25 e 75 para cada sexo em relação à silhueta corporal atual e silhueta corporal desejada estão demonstrados na Tabela 3, bem como os valores de freqüência e percentual dos indivíduos insatisfeitos com a silhueta corporal e que queiram aumentar ou diminuir a sua silhueta. As silhuetas 4 e 3 foram as mais identificadas para meninos $(36,7 \%$ e $26,6 \%)$ e meninas $(33,8 \%$ e $32,3 \%)$. As silhuetas mais desejadas foram a $4(40,4 \%)$ e a $3(45,9 \%)$ para meninos e meninas, respectivamente. Há diferenças entre os sexos nos valores de silhueta atual $(U=5996 ; p=0,016)$ e silhueta desejada $(U=3557 ; p=0,0001)$. Essa diferença pode ser observada quando analisados os valores da razão entre a silhueta corporal desejada e a silhueta corporal atual, e verifica-se que as moças apresentam maior insatisfação e tendem a querer ter uma silhueta menor que a sua silhueta atual. Analisando a diferença entre a silhueta atual e silhueta desejada de acordo com o sexo, constata-se que houve diferenças significativas somente entre as meninas $(p=0,001)$. Nesse sentido, os meninos demonstram tanto o desejo de diminuir a silhueta quanto de aumentar $(p=0,253)$. Entretanto, para as meninas, há uma tendência de desejo de diminuir a silhueta, como fica explicito na Tabela 3.

Tabela 3. Mediana, moda e percentis 25 e 75 (P25 e P75) dos valores de silhueta corporal atual, silhueta corporal desejada e a razão entre a silhueta corporal desejada e a silhueta corporal atual; freqüência e percentual dos indivíduos insatisfeitos e que queiram aumentar ou diminuir a silhueta corporal segundo o sexo.

\begin{tabular}{|c|c|c|c|c|c|c|c|c|}
\hline & Rapazes & & & & Moças & & & \\
\hline & Mediana & Moda & $P 25$ & P75 & Mediana & Moda & $P 25$ & P75 \\
\hline $\begin{array}{l}\text { Silhueta Corporal } \\
\text { Atual }\end{array}$ & $4^{*}$ & 4 & 3 & 5 & 4 & 4 & 3 & 4 \\
\hline $\begin{array}{l}\text { Silhueta Corporal } \\
\text { Desejada }\end{array}$ & $4^{*}$ & 4 & 4 & 5 & 3 & 3 & 3 & 4 \\
\hline \multirow{2}{*}{$\begin{array}{l}\text { Razão Silhueta } \\
\text { Desejada e } \\
\text { Silhueta Atual }\end{array}$} & $1,0 *$ & 1,0 & 0,8 & 1,2 & 0,83 & 1,0 & 0,75 & 1,2 \\
\hline & \multicolumn{2}{|l|}{$\mathrm{n}$} & \multicolumn{2}{|c|}{$\%$} & \multicolumn{2}{|l|}{$\mathrm{n}$} & \multicolumn{2}{|c|}{$\%$} \\
\hline $\begin{array}{l}\text { Insatisfeito com a } \\
\text { Silhueta Corporal }\end{array}$ & \multicolumn{2}{|l|}{75} & \multicolumn{2}{|c|}{69} & \multicolumn{2}{|c|}{102} & \multicolumn{2}{|c|}{76,7} \\
\hline $\begin{array}{l}\text { Aumentar } \\
\text { Silhueta }\end{array}$ & \multicolumn{2}{|l|}{44} & \multicolumn{2}{|c|}{58,7} & \multicolumn{2}{|l|}{34} & \multicolumn{2}{|c|}{33,7} \\
\hline Diminuir Silhueta & \multicolumn{2}{|l|}{31} & \multicolumn{2}{|c|}{41,3} & \multicolumn{2}{|l|}{67} & \multicolumn{2}{|c|}{66,3} \\
\hline
\end{tabular}

$p<0,05$ entre sexos
A Tabela 4 apresenta os valores de mediana, percentil 25 e 75 do nível de atividade física de meninos e meninas de acordo com o grau de satisfação em relação à silhueta corporal. Não houve associação significativa entre satisfação com a silhueta corporal e nível de atividade física em meninos $\left(x^{2}=0,809 ; g l=1 ; p=0,497\right)$ e meninas $\left(x^{2}=0,505\right.$; $g l=1 ; p=0,537)$.

Tabela 4. Mediana, percentil 25 e 75 do nível de atividade física de rapazes e moças de acordo com o módulo do grau de satisfação em relação à silhueta corporal.

\begin{tabular}{lcccccc}
\hline \multicolumn{6}{c}{ Nível de Atividade Física } \\
\hline Grupo & Rediana & $P 25$ & $P 75$ & Mediana & $P 25$ & $P 75$ \\
& 16,5 & 11,8 & 24,3 & 12 & 6 & 17 \\
Satisfeitos & 14 & 10 & 20 & 13 & 9 & 19 \\
Insatisfeitos & \multicolumn{1}{c}{ Moças } \\
\hline
\end{tabular}

\section{Discussão}

Esse estudo teve como objetivo descrever a insatisfação corporal e sua relação com o nível de atividade física habitual em adolescentes de escolas públicas de uma região urbana do município de Florianópolis. Para isso, foram utilizados instrumentos que, apesar de amplamente disseminados na literatura internacional, não possuem evidências de validade determinada para a população brasileira. Em relação ao questionário de atividade física, quando da realização da coleta do estudo, ainda não havia estudos de validação dessa variável para a população aqui estudada, podendo ser destacado ainda que o primeiro questionário validado para a população brasileira foi publicado recentemente (Florindo \& cols., 2006). Em relação às figuras de silhueta corporal, ressalta-se a tentativa de validação realizada já destacada anteriormente e a validação dessas mesmas figuras na população brasileira adulta (Scagliusi \& cols., 2006). Apesar da falta de validade desses instrumentos pode ser vista como ameaças de validade interna e externa dos achados do presente estudo, os resultados apresentados convergem para o modelo teórico vigente em relação à literatura nacional e internacional.

Este é o primeiro estudo nacional em que existe a utilização de um questionário de atividade física para diagnosticar o nível de atividade física habitual dos adolescentes e também que utilize uma escala de silhueta corporal adaptada para o uso em crianças e adolescentes.

A amostra constou de adolescentes de apenas três escolas públicas de uma região urbana de Florianópolis. Isso dificulta a extrapolação desses dados para a população do município. Entretanto, o intuito inicial era de conhecer a realidade das escolas daquele bairro, localizadas próximas à universidade e integrantes da área de atuação de projetos de extensão e pesquisa. As escolas abordadas são as únicas escolas do bairro.

De acordo com os dados levantados nesse estudo e diante de suas limitações, pode-se verificar que o nível mínimo de atividade física recomendada para obtenção de benefícios para a saúde não é encontrado em uma alta parcela dessa amostra (42,9\% e 29,4\% para meninas e meninos, respectivamente). De acordo com Nahas (2003) e Pate e cols. (1995), o nível de atividade física mínima recomendada para obtenção de benefícios para a saúde deve ser de 12 pontos no instrumento em questão. Essa prevalência de adolescentes com 
baixo nível de atividade física está de acordo com um estudo epidemiológico feito por De Bem (2003), em uma amostra representativa de adolescentes de escolas estaduais do estado de Santa Catarina, no qual a prevalência de meninas e meninos pouco ativos foi de $48,28 \%$ e $29,3 \%$ respectivamente.

$\mathrm{O}$ presente estudo encontrou que as silhuetas 4 e 3 foram as mais identificadas pelos adolescentes. Em relação à silhueta desejada, os meninos assinalaram a silhueta 4 e as meninas a silhueta 3. Mciza e cols. (2005), utilizando figuras de silhuetas similares às do presente estudo, encontraram que a silhueta 4 era considerada "normal" para meninas de 9 a 12 anos. McArthur, Holbert e Pena (2005) estudaram a questão da silhueta que mais representava o ideal de atratividade em adolescentes de 12 a 19 anos de várias cidades da América Latina: Buenos Aires (Argentina), Cidade Guatemala (Guatemala), Havana (Cuba), Lima (Peru), Cidade Panamá (Panamá) e Santiago (Chile). Para os meninos, a silhueta que mais representava o ideal de atratividade foi a $3(50 \%)$, enquanto $45 \%$ das meninas escolheram a silhueta 2 . Esse estudo, entretanto, utilizou uma escala de silhueta com nove figuras, o que de certa forma pode explicar as diferenças encontradas em relação ao presente estudo.

Além disso, foi encontrado que os meninos demonstram tanto uma tendência em aumentar a sua silhueta corporal quanto em diminuir. Por sua vez, as meninas demonstram querer diminuir a sua silhueta corporal. Esses dados são semelhantes aos encontrados na literatura, que observam no adolescente um ideal de forma corporal musculosa. Assim, meninos com baixo conteúdo muscular ou com excesso de peso estarão com maior risco de insatisfação corporal (Jones, 2004; McCabe \& Ricciardelli, 2004; McCabe \& Ricciardelli, 2005). Em relação às mulheres, parece haver uma tendência para um ideal de magreza iniciado já na infância e que as acompanham até a vida adulta. Diversas pesquisas demonstram que o ideal de imagem corporal feminina, tanto em adolescentes quanto em adultas, está predominantemente voltada para a perda de peso e que isso está associado ao ideal estético feminino de magreza (Forestell, Humphrey \& Stewart, 2004; Guendouzi, 2004; Smolak, 2004).

Verifica-se, também, uma tendência de maior insatisfação corporal nas meninas. Os dados do presente estudo demonstram que $69 \%$ dos meninos e $76,7 \%$ das meninas estão insatisfeitos com a sua silhueta corporal. Esses dados são semelhantes aos apontados pela literatura tanto para adolescentes quanto para adultos, cujos resultados têm demonstrado prevalência de insatisfação com a imagem corporal entre 50 e 70\% dos indivíduos participantes (Ricciardelli, McCabe \& Banfield, 2000). São poucos os estudos realizados no Brasil que verificaram a prevalência de insatisfação corporal em crianças e adolescentes. Os achados do presente estudo são similares aos de Triches e Giugliani (2007) que verificaram uma prevalência de 63,9\% de insatisfação corporal em escolares de 8 a 10 anos de duas cidades rurais do Rio Grande do Sul. Outro estudo com base populacional verificou uma prevalência de $82 \%$ de insatisfação corporal com escolares de 8 a 11 anos da cidade de Porto Alegre (Pinheiro, citado por Triches \& Giugliani, 2007). Branco, Hilário e Cintra (2006) verificaram a associação entre o estado nutricional e a insatisfação corporal em adolescentes de 14 a 19 anos de uma escola pública da cidade de São Paulo. Nesse estudo, eles constataram que as meninas apresentaram maior insatisfação corporal que os meninos. Entretanto, eles também verificaram que as meninas possuíam uma percepção distorcida da sua real condição, sendo que $43 \%$ das avaliadas superestimaram a sua silhueta corporal. Entre os meninos parece que houve uma subestimação em 5,6\%.

Furnham e cols. (2002), utilizando figuras de silhuetas corporais para identificação da insatisfação corporal, encontraram que somente $21,1 \%$ dos meninos e $18,3 \%$ das meninas estavam satisfeitos com a sua silhueta corporal. Os maiores valores de prevalência de satisfação, principalmente para os meninos, devem-se, provavelmente, à maior abrangência de idade no presente estudo, a qual foi de até 18 anos, versus idades de 12 e 13 anos no estudo de Furnham e cols. Estudos têm demonstrado que a insatisfação corporal tende a diminuir durante a adolescência nos meninos, devido ao aumento de massa muscular do tamanho de membros, mudanças estas relacionadas à puberdade (Bearman \& cols., 2006; McCabe \& cols., 2002), e que poderiam explicar a maior prevalência de satisfação no presente estudo.

Em relação à atividade física, não foi encontrada associação com a insatisfação corporal. Contudo, observa-se um valor de mediana acima de 12 em ambos os grupos de satisfeitos e insatisfeitos, para ambos os sexos, apesar de quase metade das meninas terem sido diagnosticadas como pouco ativa. A literatura que trata dos benefícios à satisfação corporal decorrentes da prática de atividade física é bem escassa. O que se sabe é que a atividade física pode estar associada com aspectos negativos à saúde, podendo-se destacar a relação de adolescentes praticantes de esportes com o uso de esteróides anabolizantes (Cafri, van den Berg \& Thompson, 2006; Wichstrom, 2006; Wichstrom \& Pedersen, 2001) e o uso da atividade física como meio de obtenção do ideal de beleza feminino (Furnham \& cols., 2002; McCabe \& Ricciardelli, 2004).

Existem também evidências de que a atividade física pode estar associada com aspectos positivos relacionados à saúde. Em um estudo realizado por Schnohr, Kristensen, Prescott e Scharling (2005) com 12.000 indivíduos dinamarqueses, verificou-se uma associação negativa entre atividade física de lazer e insatisfação com a vida e nível de estresse mental. Outro estudo, realizado em 754 adolescentes com idades entre 15 e 19 anos em Florianópolis, identificou que um maior nível de atividade física estava associado com menores níveis de estresse (De Bem, 2003). A atividade física, portanto, poderia servir como um promotor de maior satisfação com a vida e, conseqüentemente, com o próprio corpo.

Somente um estudo experimental averiguou a eficácia da atividade física para a redução da insatisfação corporal em adolescentes (Burgess, Grogan \& Burwitz, 2006). Esse estudo encontrou que seis semanas de treino de dança aeróbica foram capazes de diminuir a insatisfação corporal e aumentar a percepção positiva do próprio corpo em meninas escolares de 13 e 14 anos. Outros estudos em pré-adolescentes (Gehrman, Hovell, Sallis \& Keating, 2006) e adultos (Fallon \& Hausenblas, 2005) têm demonstrado resultados conflitantes em relação à eficácia da atividade física para o tratamento da insatisfação corporal.

As pesquisas sobre a insatisfação corporal em adolescentes têm demonstrado que são diversos os fatores que levam o indivíduo a estar insatisfeito com o seu corpo. Entre eles, 
pode-se citar: internalização do ideal de beleza corporal (Durkin \& Paxton, 2002); influências do próprio meio em que o adolescente convive, incluindo pessoas próximas, como familiares, amigos e outras pessoas que fazem parte do cotidiano (Bearman \& cols., 2006; Paquette \& Raine, 2004; Schwartz, Phares \& Tantleff-Dunn, 1999); e índice de massa corporal (Jones, 2004; Presnel \& cols., 2004). Dessa forma, as mudanças corporais decorrentes da puberdade e as influências de pessoas próximas e da mídia fazem da adolescência um período crítico para o desenvolvimento da insatisfação corporal (Bearman \& cols., 2006; Jones, 2004; Labre, 2002).

O ser humano tem como necessidade essencial se socializar e conviver em grupos. É evidente, portanto, a tendência de sofrer diversas influências ao querer ser aceito em determinado grupo. Na adolescência, esse processo tem, na maioria das vezes, uma maior magnitude. O ponto a ser discutido é o indivíduo se sentir pressionado a adotar um ideal de atratividade e de forma corporal que muitas vezes está longe de ser alcançado, e que pode lhe ocasionar a aquisição de uma série de comportamentos enviesados e de risco. A dependência de atividade física já foi demonstrada como uma consequiência da insatisfação corporal (McCabe \& Ricciardelli, 2004), bem como o uso de esteróides anabolizantes está associado com a prática esportiva (Cafri \& cols., 2006; Wichstrom, 2006; Wichstrom \& Pedersen, 2001). Dessa forma, a prática de atividade física que deveria trazer benefícios à saúde acaba associada com aspectos negativos relacionados à saúde.

Para uma amostra de estudantes adolescentes de escolas públicas de um bairro de Florianópolis, demonstrou-se que a maioria deles está insatisfeita com a sua silhueta corporal, sendo as meninas mais insatisfeitas do que os meninos. As meninas apresentam uma tendência maior de desejo de diminuir a silhueta corporal, enquanto os meninos demonstram tanto o desejo de aumentar quanto o de diminuir sua silhueta. Esses achados corroboram com estudos nacionais que apresentam delineamento populacional (Branco, Hilário \& cols., 2006; Triches \& Giugliani, 2007), bem como com os achados de estudos realizados em outros países (Ricciardelli \& McCabe, 2001). Tal amostra também apresenta bons níveis de atividade física em ambos os sexos, embora haja uma elevada prevalência de meninos e meninas insuficientemente ativas para obter benefícios para a sua saúde. Além disso, os meninos demonstraram ser mais ativos fisicamente do que as meninas, principalmente em atividades de lazer. Não foi encontrada associação entre insatisfação corporal e nível de atividade física

Sugere-se a realização de pesquisas que possam demonstrar a influência de outros fatores na insatisfação corporal e o incremento do número de estudos longitudinais na tentativa de obter evidências mais fortes sobre a associação entre atividade física e insatisfação corporal e de estudos experimentais para averiguar a eficácia da atividade física no tratamento da insatisfação corporal em adolescentes.

\section{Referências}

Beato-Fernández, L., Rodriguez-Cano, T., Belmonte-Llario, A. \& Martinez-Delgado, C. (2004). Risk factors for eating disorders in adolescents: A Spanish community-based longitudinal study. European Child and Adolescent Psychiatry, 13, 287-294.
Beato-Fernández, L. \& Rodriguez-Cano, T. (2005). Gender diferences regarding psychopathological, family and social characteristics in adolescents with abnormal eating behavior. Eating Behaviors, 6, 337-344.

Bearman, S. K., Martinez, E. \& Stice, E. (2006). The skinny on body dissatisfaction: a longitudinal study of adolescent girls and boys. Journal of Youth and Adolescence, 35, 217-229.

Branco, L. M., Hilário, M. O. E. \& Cintra, I. P. (2006). Percepção e satisfação corporal em adolescentes e a relação com seu estado nutricional. Revista de Psiquiatria Clínica, 33, 292-296.

Burgess, G., Grogan, S. \& Burwitz, L. (2006). Effects of a 6-week aerobic dance intervention on body image and physical selfperceptions in adolescent girls. Body Image, 3, 57-66.

Cafri, G., van den Berg, P. \& Thompson, J. K. (2006). Pursuit of muscularity in adolescent boys: relations among biopsychosocial variables and clinical outcomes. Journal of Clinical Child and Adolescent Psychology, 35, 283-291.

Childress, A. C., Brewerton, T. D., Hodges, E. L. \& Jarrel, M. P. (1993). The Kids'Eating Disorders Survey (KEDS): A Study of Middle Scholl Students. Journal of the American Academy of Child and Adolescent Psychiatry, 32, 843-850.

De Bem M. F. L. (2003). Estilo de vida e comportamentos de risco de estudantes trabalhadores do ensino médio de Santa Catarina, Tese de Dotorado, Universidade Federal de Santa Catarina, Porto Alegre.

Durkin, S. J. \& Paxton, S. J. (2002). Predictors of vulnerability to reduced body image satisfaction and psychological wellbeing in response to exposure to idealized female media images in adolescent girls. Journal of Psychosomatic Research, 53, 995-1005,

Fallon, E. A. \& Hausenblas, H. A. (2005). Media images of the "ideal" female body: can acute exercise moderate their psychological impact? Body Image, 2, 62-73.

Florindo, A. A., Romero, A., Peres, S. V., Slater, B. (2006). Development and validation of a physical activity assessment questionaire for adolescents. Revista de Saúde Pública, 40, 802-809.

Forestell, C. A., Humphrey, T. M. \& Stewart S. H. (2004). Involvement of body weight and shape factors in ratings of attractiveness by women: a replication and extension of Tassinary and Hansen. Personality and Individual Differences, 36, 295-305.

Furnham, A., Badmin, N. \& Sneade, I. (2002). Body image dissatisfaction: gender differences in eating attitudes, self-esteem, and reasons for exercise. The Journal of Psychology, 136, 581-596.

Gehrman, C. A., Hovell, M. F., Sallis, J. F. \& Keating, K. (2006). The effects of a physical activity and nutrition intervention on body dissatisfaction, drive for thinness, and weight concerns in pre-adolescents. Body Image, 3, 345-351.

Grabe, S. \& Hyde, J. S. (2006). Ethnicity and body dissatisfaction among women in the United States: a meta-analysis. Psychological Bulletin, 132, 622-640.

Guendouzi, J. (2004). 'She's very slim': talking about body-size in all-female interactions. Journal of Pragmatics, 36, 1635-1653.

Johnson, F. \& Wardle, J. (2005). Dietary restraint, body dissatisfaction, and psychological distress: a prospective analysis. Journal of Abnormal Psychology, 114, 119-125.

Jones, D. C. (2004). Body image among adolescent girls and boys: a longitudinal study. Developmental Psychology, 40, 823-835.

Kimm, S. Y. S., Glynn, N. W., Obarzanek, E., Kriska, A. M., Daniels, S. R., Barton, B. A. \& Liu, K. (2005). Relation between the changes 
in physical activity and body-mass index during adolescence: a multicentre longitudinal study. Lancet, 366, 301-307.

Labre M. A. M. P. (2002). Adolescents boys and the muscular male body ideal. Jounal of Adolescent Health, 30, 233-242.

McArthur, L. H., Holbert, D. \& Pena, M. (2005). An exploration of the attitudinal and perceptual dimensions of body image among male and female adolescents from six Latin American cities. Adolescence, 160, 801-816.

McCabe, M. P. \& Ricciardelli, L. A. (2004). A longitudinal study of pubertal timing and extreme body change behaviors among adolescent boys and girls. Adolescence, 39, 145-166.

McCabe, M. P. \& Ricciardelli, L. A. (2005). A prospective study of pressures from parents, peers, and the media on extreme weight change behaviors among adolescent boys and girls. Behaviour Research and Therapy, 43, 653-668.

McCabe, M. P., Ricciardelli, L. A. \& Finemore, J. (2002). The role of puberty, media and popularity with peers on strategies to increase weight, decrease weight and increase muscle tone among adolescent boys and girls. Journal of Psychosomatic Research, 52, 145-153.

Mciza, Z., Goedecke, J. H., Steyn, N. P., Charlton, K., Puoane, T., Meltzer, S., Levitt, N. S. \& Lambert, E. V. (2005). Development and validation of instruments measuring body image and body weight dissatisfaction in South African mothers and their daughters. Public Health Nutrition, 8, 509-519.

McKinley, N. M. (1999). Women and objectified body consciousness: mothers' and daughters' body experience in cultural, developmental, and familial context. Developmental Psychology, 35, 760-769.

Nahas, M. V. (2003). Atividade física, saúde e qualidade de vida. Londrina: Midiograf.

Paquette, M. \& Raine, K. (2004). Sociocultural context of women's body image. Social Science and Medicine, 59, 1047-1058.

Pate, R. R., Pratt, M., Blair, S. N., Haskel, W. L., Macera, C. A., Bouchard, C., Buchner, D., Ettinger, W., Heath, G. W. \& King, A. C. (1995). Physical activity and public health: A recommendation from the Centers for Disease Control and Prevention and the American College of Sports Medicine. The Journal of the American Medical Association, 273, 402-407.

Pedersen, B. K. \& Saltin, B. (2006). Evidence for prescribing exercise as therapy in chronic disease. Scandinavian Journal of Medicine \& Science in Sports, 16, 3-63.

Presnell, K., Bearman, S. K. \& Stice, E. (2004). Risk factors for body dissatisfaction in adolescent boys and girls: a prospective study. International Journal of Eating Disorders, 36, 399-401.

Ricciardelli, L.A. \& McCabe, M. (2001). Children's body image concerns and eating disturbance: a review of the literature. Clinical Psychology Review, 21, 325-344.

Ricciardelli, L. A., McCabe, M. P. \& Banfield, S. (2000). Body image and body changes methods in adolescent boys. Role of parentals, friends, and the media. Journal of Psychosomatic Research, 49, 189-197.

Rodriguez-Cano, T., Beato-Fernández, L. \& Llario, A. B. (2006). Body dissatisfaction as a predicter of self-reported suicide attempts in adolescents: A Spanish community prospective study. Journal of Adolescent Health, 38, 684-688.
Scagliusi, F. B., Alvarenga, M., Polacow, V. O., Cordás, T. A., Queiroz, G. K. O., Coelho, D., Philippi, S. T. \& Lancha Jr, A. H. (2006). Concurrent and discriminate validity of the Stunkard's figure rating scale adapted into Portuguese. Appetite, 47, 77-82.

Schnohr, P., Kristensen, T. S., Prescott, E. \& Scharling, H. (2005). Stress and life dissatisfaction are inversely associated with jogging and other types of physical activity in leisure timeThe Copenhagen City Heart Study. Scandinavian Journal of Medicine and Science in Sports, 15, 107-112.

Schwartz, D. J., Phares, V., Tantleff-Dunn S. \& Thompson, J. K. (1999). Body image, psychological functioning, and parental feedback regarding physical appearance. International Journal of Eating Disoders, 25, 339-343.

Smolak, L. (2004). Body image in children and adolescents: where do we go from here? Body image, 1, 15-28.

Smolak, L., Levine, M. P. \& Thompson, J. K. (2001). The use of the Sociocultural Attitudes Towards Appearance Questionnaire with middle school boys and girls. International Journal of Eating Disorders, 29, 216-223.

Stice, E. \& Bearman, S. K. (2001). Body image and eating disturbances prospectively predict increases in depressive symptoms in adolescent girls: a growth curve analysis. Developmental Psychology, 37, 597-607.

Stice, E. \& Shaw, H. E. (2002). Role of body dissatisfaction in the onset and maintenance of eating pathology: a synthesis of research findings. Journal of Psychosomatic Research, 53, 985-993.

Stice, E. \& Whitenton, K. (2002). Risk factors for body dissatisfaction in adolescent girls: a longitudinal investigation. Developmental Psychology, 38, 669-678.

Stunkard, A. J., Sorensen, T. \& Schalsinger, F. (1983). Use of the Danish adoption register for the study of obesity and thinness. Genetics of Neurological and Psychiatric Disorders, 60, 115-120.

The McKnight Investigators (2003). Risk factors for the onset of eating disorders in adolescent girls: results of the McKnight Longitudinal Risk Factor Study. American Journal of Psychiatry, 160, 248-254.

Thomas, J. R. \& Nelson J. K. (2002). Métodos de pesquisa em atividade física. Porto Alegre: Artmed.

Triches, R. M. \& Giugliani, E. R. J. (2007). Insatisfação corporal em escolares de dois municípios da região sul do Brasil. Revista de Nutrição, 20,119-128.

Van Strien, T., Engels, R. C. M. E., Leeuwe, J. V. \& Snoek, H. M. (2005). The Stice model of overeating: tests in clinical and non-clinical samples. Appetite, 45, 205-213.

Wichstrom, L. (2006). Predictors of future anabolic androgenic steroid use. Medicine \& Science in Sports \& Exercise, 38, 1578-1583.

Wichstrom, L. \& Pedersen, W. (2001). Use of anabolic-androgenic steroids in adolescence: winning, looking good or being bad. Journal of Studies on Alcohol, 62, 5-13.

Recebido em 21.03.2007

Primeira decisão editorial em 24.07.2007

Versão final em 24.08.2007

Aceito em 30.11.2007 